\title{
Influencia de la estructura urbana en los patrones de movilidad cotidiana de un trabajo feminizado en la Zona Metropolitana de la Ciudad de México
}

\section{INFLUENCE OF THE URBAN STRUCTURE ON THE PATTERNS OF DAILY MOBILITY OF A FEMINIZED WORK IN THE METROPOLITAN AREA OF MEXICO CITY}

\author{
INFLUÊNCIA DA ESTRUTURA URBANA NOS PADRÕES DE MOBILIDADE \\ COTIDIANA DE UM TRABALHO FEMINIZADO NA REGIÃO \\ METROPOLITANA DA CIDADE DO MÉXICO
}

JOSEFINA HERNÁNDEZ Lozano ${ }^{1}$

Hernández Lozano, J. (2018). Influencia de la estructura urbana en los patrones de movilidad cotidiana de un trabajo feminizado en la Zona Metropolitana de la Ciudad de México. Perspectiva Geográfica, 23(2). 10.19053/01233769.7380

\section{Recepción:}

14 de octubre de 2017

Evaluación:

27 de abril de 2018

Aprobación:

7 de mayo de 2018

\section{Resumen}

Una de las consecuencias de la estructura urbana segregada es el incremento en las distancias de desplazamiento entre el centro y la periferia. Sus efectos no han sido homogéneos en todo el territorio. Para el análisis de la distribución de los patrones de movilidad de una ocupación altamente feminizada como el trabajo doméstico remunerado, se optó por el análisis exploratorio de datos espaciales, aplicando el índice de Moran y el índice local de autocorrelación espacial (LISA). Los resultados obtenidos muestran una mayor movilidad externa de las mujeres, con largos desplazamientos, tanto en distancia como en tiempo, desde municipios del oriente de la Zona Metropolitana de la Ciudad de México, que se caracterizan por tener altos niveles de pobreza.

1 Licenciada en Geografía de la Universidad Nacional Autónoma de México. Correo electrónico: hlozano@unam.mx. 
Palabras clave: Ciudad de México, género, movilidad cotidiana, segregación espacial y trabajo doméstico remunerado.

\section{Abstract}

One of the consequences of segregated urban structure is the increase in the distances of displacement between the center and the periphery. Its effects have not been homogeneous throughout the territory. For the analysis of the distribution of the commuting patterns of a highly feminized occupation such as paid domestic work, we opted for the exploratory analysis of spatial data, applying the Moran Index and the Local Autocorrelation Index Space (LISA). The results obtained show a greater external mobility of women, with long distances both in distance and time, from municipalities in the eastern zone of the Metropolitan Area of Mexico City, which are characterized by having high levels of poverty.

Keywords: Mexico City, gender, commuting, spatial segregation and paid domestic work.

\section{Resumo}

Uma das conseqüências da estrutura urbana segregada é o aumento das distâncias de deslocamento entre o centro e a periferia. Seus efeitos não foram homogêneos em todo o território. Para a análise da distribuição dos padrões de mobilidade de uma ocupação altamente feminizada, como o trabalho doméstico remunerado, optamos pela análise exploratória de dados espaciais, aplicando o índice de Moran e o índice de autocorrelação local espaço (LISA). Os resultados obtidos mostram uma maior mobilidade externa das mulheres, com deslocamentos longos tanto na distância quanto no tempo, dos municípios da região leste da Área Metropolitana da Cidade do México, caracterizados por altos níveis de pobreza.

Palavras-chave: Cidade do México, gênero, mobilidade diária, segregação espacial e trabalho doméstico remunerado. 


\section{Introducción}

El acceso a la ciudad y a sus diferentes servicios está determinado en parte por la infraestructura del transporte, y esta, a su vez, por la estructura urbana de la ciudad. Así mismo, el grado de expansión urbana va a influir en los límites que alcance la ciudad y en los tiempos de viaje entre el centro y la periferia. La configuración de la estructura urbana metropolitana y su organización funcional suponen una dependencia de los habitantes de la periferia respecto del centro de la metrópoli (Avellaneda, 2009). Así es que en un contexto de segregación espacial la población ocupada presenta diferentes patrones de comportamiento frente a la movilidad. Los trabajadores se desplazan a sus actividades desde ámbitos geográficos diversos y con patrones diferenciados; por ejemplo, según el género, se considera que las mujeres se desplazan para trabajar a distancias más cortas de su lugar de residencia, a diferencia de los hombres (Casado, 2000; Blumen, 1994).

Blumen (1994) reconoce tres factores que determinan que las distancias de desplazamiento sean más cortas para las mujeres: la residencia, el empleo y el transporte; estos factores contienen tanto aspectos sociales como espaciales. En el caso de las mujeres casadas, el aspecto que determina las distancias cortas de desplazamiento residencia-trabajo son sus responsabilidades familiares y domésticas; su empleo tiende a ser a tiempo parcial o por horas y sus viajes pueden ser más a pie o en transporte público. Así mismo, identifica que las diferencias de género en los desplazamientos casi siempre son mayores en la periferia, en comparación con la ciudad central. Barrios y Hernández (2014) afirman que, además del género, la capacidad de desplazamiento es diferente según sea la edad, el estado civil, el nivel de estudios y la rama de actividad en la que se trabaje.
Particularmente el estudio de las mujeres desde una perspectiva que incorpora geografía y género enfatiza cómo la diferencia de género se traduce en desigualdad espacial (Jirón y Zunino, 2017).

La movilidad cotidiana, abordada desde una perspectiva de geografía de género, hace posible distinguir realidades enmascaradas por los enfoques tradicionales, que han tenido un carácter más agregado, lo que ha llevado a asumir patrones comunes para toda la población (Díaz, 1989). La geografía de género permite hacer evidentes las diferencias existentes entre hombres y mujeres y revelar dichas diferencias en el espacio con patrones particulares.

El propósito fundamental de este trabajo es identificar los patrones de movilidad de un trabajo feminizado en una estructura urbana segregada. Precisamente una de las ocupaciones más feminizadas es el trabajo doméstico remunerado (TDR), ocupación identificada con una movilidad predominantemente de "cercanía" (Susino y Torrado, 2015). El TDR es una ocupación informal con alta precariedad laboral, que se caracteriza por bajos salarios y poca o nula cobertura de seguridad social, tienen como fuente de empleo principalmente las unidades domésticas, es decir, hogares de los sectores de ingreso medio y alto, en donde la inserción laboral de las mujeres genera una demanda por el servicio, y con una localización dominante al poniente de la ciudad, ejemplo de la segregación espacial que caracteriza a la Zona Metropolitana de la Ciudad de México (ZMCM). A partir de lo anterior es posible plantear las siguientes preguntas: ¿cuál es el efecto de la segregación urbana sobre los patrones de movilidad cotidiana de los trabajadores domésticos remunerados en la ZMCM?, ¿existe alguna diferencia en la movilidad cotidiana según género?, ¿se emplean solo en su entorno local o 
realizan desplazamientos de larga distancia a su lugar de trabajo? Se tratará de responder a estas preguntas en los párrafos siguientes.

\section{Estructura urbana, movilidad y diferencia de género}

La expansión urbana dispersa o "ciudad dispersa", característica de las ciudades latinoamericanas, se extiende de forma horizontal sobre el territorio, principalmente con periferias de tipo expandido y fragmentado; comprende bajas densidades, discontinuidades y segregación espacial que tienden a incrementar los desplazamientos y los flujos de movilidad entre centro-periferia (Pradilla, 2015). Los desarrollos habitacionales se realizan al borde de la trama urbana compacta de la ciudad (Susino y Duque, 2013), por lo que para Duhau y Giglia (2008) el crecimiento periférico de las metrópolis transitó del modelo de ciudad compacta hacia un modelo urbano difuso y sin límites definidos. El poblamiento de la periferia, resultado de la expansión dispersa, se presenta en áreas cada vez más alejadas que se incorporan a la zona metropolitana y en algunos casos se caracterizan por concentrar población de bajos ingresos, ser zonas carentes de los servicios urbanos básicos y con deficiente infraestructura de transporte. Estas zonas con similar condición socioeconómica, situadas en la periferia lejana, definen claramente la segregación espacial de las ciudades latinoamericanas y son producto del mercado del suelo, que ha condicionado sis-

2 El primer concepto de ciudad dispersa fue descrito por Earl Draper en 1937 (Nechyba et al., 2004 citado por Gielen, 2016) y desde entonces se ha vuelto popular, aunque actualmente todavía es difícil encontrar consenso en su definición. Galster et al. (2001, citados por Gielen, 2016) definen la ciudad dispersa como: "Sprawl (n.) is a pattern of land use in UA (unit of analysis) that exhibits low levels of some combination of eight distinct dimensions: density. continuity, concentration, clustering, centrality, nuclearity, mixed use, and proximity" (p. 685) temáticamente a la población de bajos ingresos a concentrarse en el suelo urbano de menor precio y con las peores condiciones ambientales (López, 2015). Para Jaramillo (1999), la relación entre mercado del suelo y estructura urbana ha sido clave en la formación de algunos rasgos socioespaciales comunes en las ciudades latinoamericanas, como la suburbanizacion de las elites y la segregación socioespacial. La expansión urbana conforma así una periferia excluyente con colonias populares en donde se presenta un proceso de segregación funcional por tener un carácter principalmente residencial, pues a pesar de que adquieran funciones urbanas con su consolidación no llegan a considerarse como lugares con diversidad funcional (Avellaneda, 2008). Son lugares sin dotación de servicios básicos y con pocas oportunidades de empleo, de modo que la movilidad se vuelve una necesidad obligada para su integración a la ciudad. En resumen, la dinámica urbana está conformada por dos elementos con un vínculo innegable: el lugar de residencia y el lugar de trabajo (o de estudio), que tienen en la movilidad cotidiana el elemento de conexión. Por su parte, la extensión territorial es un importante componente espacial que determina las diferentes formas de interacción residencia-trabajo y principalmente los flujos de movilidad cotidiana.

La movilidad cotidiana ${ }^{3}$, pendular o commuting se entiende como la suma de los desplazamientos que realiza la población de forma recurrente para acceder a bienes y servicios en un territorio determinado (Miralles-Guasch, 1998, citado por Miralles y Cebollada, 2009). Es así como la movilidad cotidiana juega un papel muy importante en los

3 En este artículo los términos movilidad, desplazamiento y movilidad cotidiana se empelan indistintamente. 
procesos de segregación residencial. La población que reside en la periferia de la ciudad encuentra más dificultad para desplazarse por los problemas para conseguir un medio de transporte eficiente y económico. Para la Ciudad de México se considera que un $34 \%$ de los desplazamientos requieren de uno o más transbordos (Moovit, 2016) debido a las largas distancias que deben recorrer entre el lugar de residencia y el centro de trabajo.

De acuerdo con Tarrius (1978 citado por Lazo y Contreras, 2009), la movilidad no se define por la suma de desplazamientos individuales ni por la accesibilidad ni la unión de puntos en el espacio urbano, sino que más bien corresponde a un fenómeno social, determinado por relaciones sociales, necesidades y modos de vida. Así mismo, se ha hecho evidente en la literatura un cambio en el foco de interés, que ha pasado de los medios de transporte hacia las personas y la movilidad cotidiana, es decir, a las personas que usan la infraestructura y los servicios de transporte (Moreno y Rubiano, 2014). La movilidad se convierte entonces en un indicador de los procesos de cambio territorial y afecta de forma diferente a los distintos grupos de población (Rodríguez y García, 2012) debido a posibles limitaciones temporales, físicas, económicas o funcionales. En términos generales, la movilidad presenta diferencias por género y estrato socioeconómico (Díaz, 1989).

Como señalan Rodríguez y García (2012), el cruce entre movilidad y género, como perspectiva de análisis, hace visible un conjunto de desigualdades en el acceso a las oportunidades de la vida en la ciudad. En particular, se ha demostrado que los patrones de movilidad de las mujeres, debido a sus roles de género en la estructura social, tienen características distintas; las mujeres, en general, viven más cerca de su lugar de trabajo y tienen la posibilidad de ir andando y de utilizar el transporte público (Miralles-Guasch y Martínez-Melo, 2012). En otros estudios se ha enfatizado que las mujeres casadas se desplazan más cerca de su lugar de residencia que las mujeres solteras y que los hombres, independientemente del estado civil de estos (Valdés, 2008; Blumen, 1994). Adicionalmente, se ha identificado que en estratos socioeconómicos bajos los desplazamientos de corta extensión de las mujeres suelen ser más pedestres, lo cual delimita su radio de acción a los barrios y su entorno inmediato (Figueroa y Waintrub, 2015), pero se olvida que el nivel de ingresos también determina su capacidad de desplazarse (Jouffe, 2011).

El interés de la geografía de género por interpretar el espacio urbano según el género encuentra en la movilidad urbana unas de las principales preocupaciones desde los años noventa (Soto, 2017). La geografía de género tiene como objetivo reconocer la forma en que las diferencias entre hombres y mujeres tienen una traducción espacial y descubrir el peso del espacio como medio en el que se plasman (Díaz, 1995). Los temas de geografía de género en México inician en la década de los noventa con asuntos como el mercado de trabajo y se diversifican posteriormente hacia la participación política, la violencia hacia las mujeres, la relación entre ciudad y género, y se destaca el tema de turismo sexual, sin olvidar las biografías de geógrafas (Ibarra y Escamilla, 2016); sin embargo, a pesar de lo anterior, el género es un tema todavía con una presencia reciente en la geografía nacional y con gran potencial de desarrollo. 


\section{3. Ámbito de estudio, metodología y datos}

La Zona Metropolitana de la Ciudad de México (ZMCM) se ha caracterizado por su expansión desordenada y una urbanización desprovista de infraestructura y servicios en la periferia de su territorio. Como en varias ciudades de América Latina, el proceso de urbanización se ha basado en invasiones o loteos irregulares desarrollados en la periferia, en suelo accesible a los pobres, que regularmente carece de infraestructura básica (Pírez, 2013). Estudios recientes coinciden y confirman un patrón de diferenciación oriente/ poniente en la ZMCM y señalan la concentración de los estratos altos y medio altos hacia la zona poniente y sur poniente, en contraste con los estratos bajos y muy bajos, presentes hacia la zona oriente (Aguilar, Romero y Hernández, 2015). Por su parte, Graizbord y Acuña (2007) plantean que es posible dividir la ciudad en "rica" poniente y ciudad "pobre" oriente a partir de identificar que en esta segunda zona predomina una población con ingresos relativamente inferiores al promedio del área metropolitana.
Actualmente se considera que la zona oriente de la ZMCM ha presentado el más intenso proceso de crecimiento urbano, con una composición desordenada de vivienda, industria y servicios; constituye el área más grande en donde se ubican las colonias populares con los niveles de ingresos más bajos (Moreno y Partida, 2011). Esta distribución hace evidente el proceso de segregación socioespacial, lo que acentúa la desigualdad urbana y determina patrones de movilidad. Según los datos de situación de pobreza por municipio del Consejo Nacional de Evaluación de la Política de Desarrollo Social (Coneval, 2010) (Tabla 1), se reportan porcentajes de $19,5 \%$ para la ciudad central y $47,2 \%$ para los municipios conurbados de la zona oriente. Respecto al porcentaje de población en la categoría de “ingreso inferior a la línea de bienestar", la ciudad central tiene un porcentaje de $24,2 \%$ y los municipios conurbados de la zona oriente un porcentaje de $50,8 \%$. La distribución de la pobreza destaca en la zona oriente, en los municipios de Chimalhuacán y Valle de Chalco, con un porcentaje de 62,7 y 58,6 respectivamente (Figura 1).

\section{TABLA 1. PORCENTAJE DE POBREZA Y SELECCIÓN DE INDICADORES, 2010}

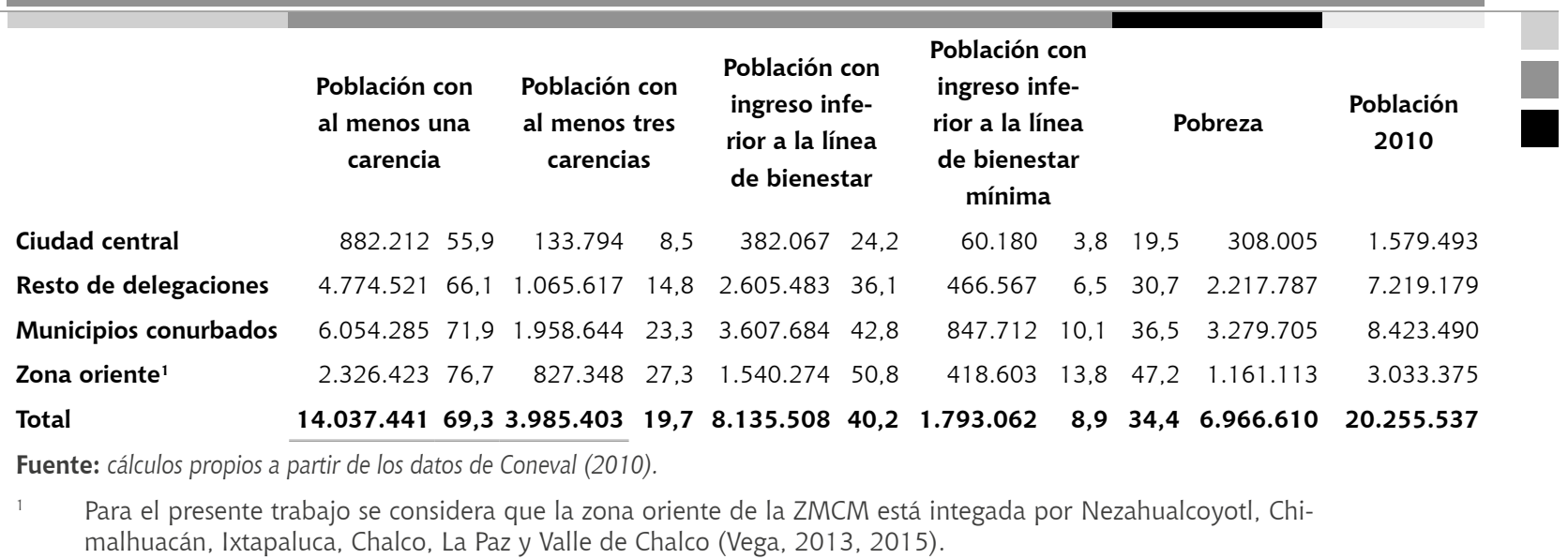




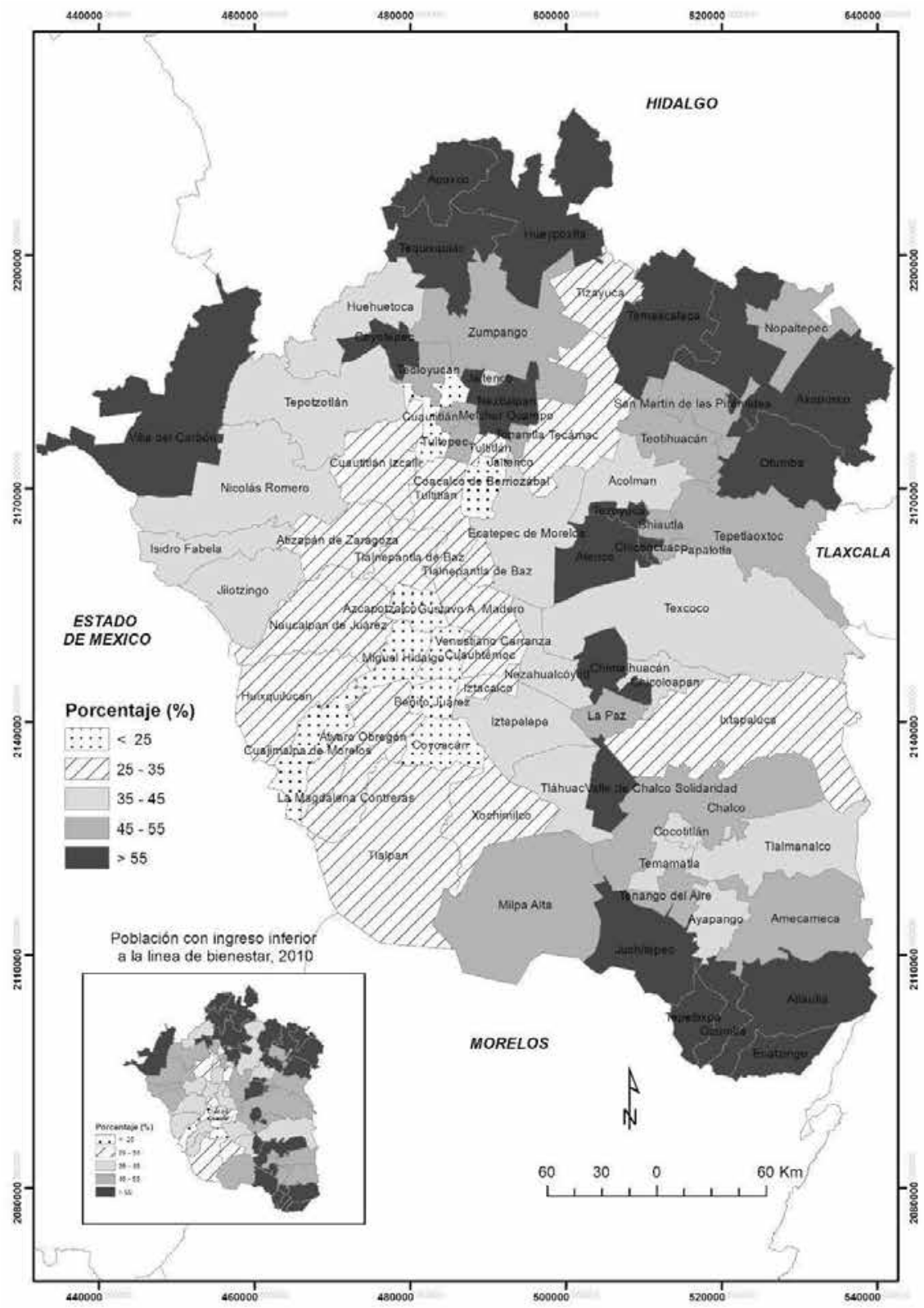

\section{FIGURA 1.}

ZMCM: Población en

Situación de Pobreza, 2010

Fuente: Coneval (2010).

Los impactos de la pobreza al interior de la ciudad son diversos, producen segregación urbana con patrones dominantes y una exclusión social con diferentes grados de intensidad (Carrión, 2003). La pobreza, entendida como la insuficiencia de recursos que impide a los que la sufren satisfacer sus necesidades esenciales, puede ser considerada como un elemento que dificulta la integración plena a la ciudad (Avellaneda, 2008).
En este trabajo se toma como ámbito de estudio la Zona Metropolitana de la Ciudad de México (ZMCM) para conocer cómo afectan las diferencias de género en la movilidad a los trabajadores domésticos remunerados, en una estructura urbana con una clara segregación espacial. Para ello se utilizaron los datos cuantitativos obtenidos de la Encuesta Intercensal (Instituto Nacional de Estadística y Geografía [INEGI], 2015a) y se buscó 
identificar los patrones de distribución de los TDR, principalmente, ubicar agrupamientos de municipios "expulsores" de este tipo de trabajadores, es decir, analizar si existe una autocorrelación espacial de la movilidad externa de los TDR. Ocasionalmente, y solo para efectos de apoyo, se usaron otras fuentes como encuestas de hogares, específicamente la Encuesta Nacional de Ocupación y Empleo (ENOE) (INEGI, 2015b).

A partir de la información de la muestra intercensal se obtuvo una matriz de movilidad a nivel municipal que permitió identificar la movilidad laboral diaria de los TDR en la ZMCM. Específicamente se pudo obtener el porcentaje de movilidad interna (MI) o población ocupada que vive y trabaja en el mismo municipio y movilidad externa (ME) o población ocupada que trabaja en un municipio distinto a donde reside.

Para identificar los patrones de movilidad del TDR, se procedió a comprobar si existía autocorrelación espacial en la distribución de la movilidad externa del trabajo doméstico remunerado. Se utilizó el análisis exploratorio de datos espaciales (AEDE) y se aplicaron los índices de Moran global (I) y Moran local (LISA). La autocorrelación espacial mide si las unidades espaciales conforman un patrón determinado o si se distribuyen aleatoriamente. En particular, los índices de asociación espacial miden en un valor la intensidad de la agrupación en toda el área de estudio (Moran global I) y, por otro lado, permiten identificar y visualizar aglomeraciones espaciales estadísticamente significativas al interior del área de estudio (Moran local LISA) (García, Tomaseti y Sánchez, 2013; Melo y De Freitas, 2010).

El mapa de LISA (por la sigla en inglés de local indicator of spatial asociation) resultante permite identificar y visualizar cartográficamente unidades espaciales donde se agrupan valores similares altos o bajos, así como atípicos espaciales, que son unidades con valores distintos a los de las áreas que las rodean. Las combinaciones espaciales que resultan a partir de los valores de las unidades son: alto-alto (denominados puntos calientes o hot spots), bajo-bajo (denominados puntos fríos o cold spots), bajo-alto, alto-bajo y relación no significativa (García et al., 2013). En este trabajo, el nivel de significancia se consideró en $p<0,05 \mathrm{y}$ el procesamiento se realizó utilizando el programa ESRI ArcGIS 10.1.

Además, considerando que la movilidad espacial es un fenómeno sensible a variables demográficas como la edad, el estado civil y el contexto familiar (Módenes, 2008), se utilizaron algunas variables para analizar las características sociodemográficas de la movilidad interna y externa de los TDR. Adicionalmente, la información del tipo de medio y la duración del traslado permitieron apoyar el análisis.

\section{El trabajo doméstico remunerado y la movilidad según género}

Los trabajadores domésticos constituyen una parte considerable de la fuerza de trabajo en empleo informal, se concentran en los estratos más bajos del ingreso y se encuentran entre los grupos de trabajadores más vulnerables. Con respecto a lo anterior, cabe mencionar que, de acuerdo con la clasificación socioeconómica del INEGI, la clase baja que agrupa los ingresos más bajos tiene un ingreso máximo al mes aproximado de 275 dólares (dos salarios mínimos) y la clase alta, por su parte, tiene un ingreso al mes mayor a 1.362 dólares (nueve salarios mínimos) (Siscar, 2015). 
Según datos de la Encuesta Nacional de Ocupación y Empleo (ENOE) para 2015, la población ocupada en el país era de 51,8 millones de personas, de las cuales 2,4 millones eran TDR, que representa el 4,7\% del total del personal ocupado en México. El conjunto de trabajadores domésticos abarca diferentes ocupaciones como: empleados domésticos, lavanderos y planchadores domésticos, choferes en casas particulares, cocineros domésticos, cuidadores de personas adultas y atención de menores (INEGI, 2012). El grupo de empleados domésticos que realiza quehaceres de limpieza en casas particulares constituye $83,9 \%$ del total de TDR y representa 1,9 millones de personas. El trabajo doméstico, además de ser una ocupación en extremo feminizada, es un fenómeno predominantemente urbano y es la principal forma de acceso al mercado del trabajo para las mujeres más pobres y con menor nivel educativo. Así mismo, se puede destacar que los tipos de tareas domésticas realizadas según género tienen una diferencia marcada: las mujeres realizan tareas de limpieza en hogares particulares, cuidadoras de adultos mayores y niños y lavanderas o planchadoras en casas particulares, mientras que los hombres principalmente son choferes, jardineros, empleados generales y cocineros en casas particulares.

Según la ENOE 2015 (INEGI, 2015b), se estima que el $11 \%$ de las mujeres ocupadas se desempeña como trabajadoras domésticas remuneradas y, de acuerdo con la Organización Internacional del Trabajo (OIT, 2013), se estima que entre el 10y el $15 \%$ de los hogares cuenta en forma estable con apoyo doméstico remunerado. Lo anterior se incrementa si se considera la contratación por día o por hora, que se realiza básicamente por familias de sectores medios. También existen familias de bajos ingresos, incluidas aquellas de las propias trabajadoras domésticas, que acuden a otras mujeres de la familia o vecinas para apoyar el trabajo del hogar.

En este trabajo se considera que la movilidad laboral cotidiana está representada por los flujos de trabajadores entre municipios distintos. La información que ofrece la Encuesta Intercensal 2015 (INEGI, 2015a) nos permite identificar la ocupación y el lugar de trabajo. En este caso, los trabajadores domésticos remunerados (TDR) presentan una movilidad del $28,8 \%$, donde un $0,6 \%$ corresponde a los hombres y un $28,2 \%$ a las mujeres. En cuanto al diferente comportamiento de ambos sexos, según grupos de edad, sobresale la mayor proporción de hombres en los grupos de edad más extremos con respecto a las mujeres: el $20 \%$ de los hombres trabajadores domésticos que se desplazan tiene 29 años o menos por solo $10,7 \%$ de las mujeres. La proporción de movilidad de hombres que tienen 65 años o más es del 15,9\%, mientras que la proporción de las mujeres que se desplaza en este rango de edad es del 4,4\%.

La diferencia de movilidad según género en función de la situación conyugal presenta la proporción más alta para hombres y mujeres en casados o unidos, con un porcentaje de 35,8 y $21,7 \%$ respectivamente para el caso de los hombres, frente al $32,3 \%$ y $22,4 \%$ para el caso de las mujeres. Respecto a la relación de parentesco de los TDR según género, se encuentra que los hombres tienen una movilidad mayor en la categoría de jefe del hogar, con un $65,3 \%$, mientras que para las mujeres en esta condición el porcentaje es del 39,5\%. La relación de parentesco más común entre los TDR es el de esposos(as), con un porcentaje más significativo en las mujeres, de $41,3 \%$, a diferencia de los hombres, con un 9,5\%.Por último, los hijos del 
jefe del hogar son la tercera relación de parentesco más significativa, correspondiente al $15,7 \%$ en los hombres y el 10,2 \% en las mujeres. En cuanto al nivel de escolaridad de hombres y mujeres TDR, la proporción de desplazamientos se concentra, en ambos casos, en el nivel de primaria y secundaria, con el 41 y el $30 \%$ para los hombres y un 48 y $34,9 \%$ para mujeres respectivamente.

Existe una mayor proporción de desplazamientos de hombres ocupados en el TDR que cuentan con un nivel de escolaridad de bachillerato (13\%), mientras que en el caso de las mujeres los porcentajes son del 6,6\%. Estas diferencias en el nivel educativo influyen en el tipo de ocupación de los TDR: los hombres con bachillerato tienden a ocuparse como choferes y las mujeres, por su parte, se ocupan como cuidadoras de personas en domicilio (INEGI, 2012). En cuanto a las variables de movilidad, hombres y mujeres presentan los porcentajes más altos en los tiempos de traslado de más de una hora y hasta dos, con 40,7 y 45,6\% respectivamente. En cuanto al medio de traslado según género, el porcentaje más alto para los dos coincide en camión, combi o colectivo, con un $89 \%$. Los TDR, tanto hombres como mujeres, residen principalmente en las delegaciones Iztapalapa y Álvaro Obregón. Por su parte, los municipios que expulsan más TDR, tanto hombres como mujeres, son Iztapalapa y Chimalhuacán. Finalmente, el máximo número de municipios a donde se desplazan tiene una diferencia importante: los hombres se desplazan, como máximo, a ocho diferentes municipios, principalmente las delegaciones de la Ciudad de México, mientras que las mujeres se desplazan a 27 diferentes municipios, incluyendo delegaciones.

\section{La diversidad en el patrón femenino de movilidad de los TDR}

Considerando la extrema feminización de los TDR, evaluamos en este trabajo su distribución espacial para identificar si la estructura urbana influye en la conformación de patrones de movilidad espacial. Recordemos que en el trabajo doméstico remunerado, tradicionalmente, se han identificado las modalidades de puertas afuera, puertas adentro o por día; estas formas de emplearse se relacionan directamente con la movilidad cotidiana. Básicamente, su movilidad abarcara desde ningún desplazamiento, al vivir en el hogar que las emplea, hasta diferentes tiempos, distancias y medios de desplazarse desde su lugar de residencia hasta el lugar de trabajo.

Otra característica importante en el trabajo doméstico remunerado se asocia al estado civil: en la modalidad puertas adentro se emplean mujeres solteras que viven en hogares de clase alta y media alta y trabajan exclusivamente para el hogar que las emplea. El empleo puertas afuera con un solo empleador es una opción para las mujeres casadas que tienen familias. Una desventaja de esta modalidad es el costo del transporte, que no se limita al costo monetario, sino también al tiempo de viaje (Rodgers, 2009).

\subsection{La modalidad de desplazamientos de los TDR y la variable espacial}

Los porcentajes de movilidad cotidiana interna reportan que 66 de los 76 municipios tienen un valor mayor al $50 \%$, donde los de mayor movilidad interna son Benito Juárez (93,4\%), seguido por Miguel Hidalgo $(96,1 \%)$ y Cuautitlán Izcalli 
(92\%). Los municipios con menor movilidad interna (MI), y, por ende, mayor movilidad externa (ME), son Chimalhuacán (59,9\%), Isidro Fabela (64,3 \%), La Paz $(69,7 \%)$ y Valle de Chalco $(63,1 \%)$. Son municipios que establecen más interrelaciones con sus vecinos, que se refleja en diferentes flujos de salidas y entradas de trabajadores. Como se puede observar, la movilidad de las TDR sigue un patrón diferente en el centro y la periferia, se presentan valores altos de MI en las delegaciones de la Ciudad Central y, a su vez, valores altos de ME en los municipios de la periferia metropolitana.

En cuanto a la distribución espacial de la ME de las TDR, se buscó identificar agrupamientos de municipios o áreas homogéneas de movilidad externa en la ZMCM. Para lo anterior se utilizó el análisis exploratorio de datos espaciales (AEDE) y se aplicaron los índices de Moran global (I) y Moran local (LISA). La autocorrelación espacial mide si las unidades espaciales conforman un patrón determinado o si se distribuyen aleatoriamente. En particular, los índices de asociación espacial miden en un valor la intensidad de la agrupación en toda el área de estudio (Moran global I) y, por otro lado, permiten identificar y visualizar aglomeraciones espaciales estadística- mente significativas al interior del área de estudio (Moran local LISA) (García et al., 2013; Melo y De Freitas, 2010). El índice de Moran global para $\mathrm{ME}$ en la zona metropolitana fue de 0,30 estadísticamente significativo ( $p<0,05)$, lo cual indica que existe autocorrelación espacial de la movilidad externa de los TDR con tendencia a la agrupación.

La distribución de la variable fue examinada en menor escala por medio del I Moran local (LISA), produciendo así un valor específico para cada municipio que permite la visualización de agrupamientos de municipios con valores similares para la variable seleccionada, en este caso, la movilidad externa de TDR. Según el índice local de Moran, que permite visualizar las agrupaciones o clústeres municipales, el 7,8 \% (6/76) de municipios se encuentran en espacios donde la relación es alto-alto, es decir, la presencia de municipios con predominio alto de ME (superior a la media) rodeados de municipios con predominio alto de ME (Figura 2). Las agrupaciones espaciales se concentraron en la zona oriente y norponiente de la zona metropolitana, que básicamente corresponde a la periferia de la zona metropolitana. 


\section{FIGURA 2.}

ZMCM: índice de Moran, 2015.

Fuente: cálculos propios a partir de la Encuesta Intercensal 2015 del INEGI.

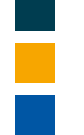
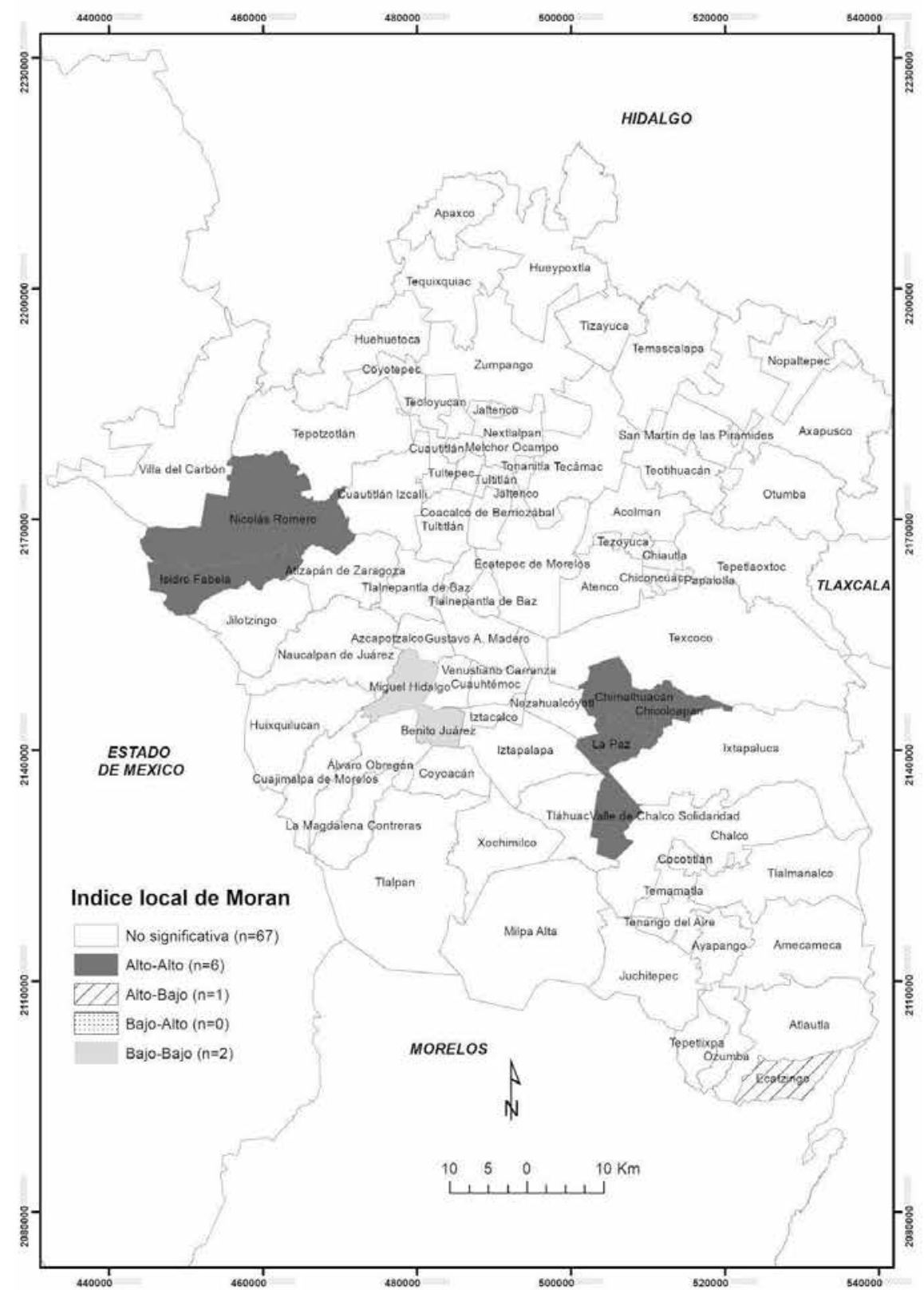

Las correlaciones del tipo bajo-bajo fueron significativas solo en el 2,6\% (2/76) y corresponden municipios con un bajo porcentaje de ME rodeados de otros con un porcentaje bajo. Esta agrupación se ubica en las delegaciones de la ciudad central Benito Juárez y Miguel Hidalgo, delegaciones que, por consiguiente, cuentan con los valores más altos de MI.
Estos patrones o modalidades diferentes de movilidad tienen sentido si consideramos que las TDR se emplean básicamente en hogares de los sectores de ingreso medio y alto, grupos que predominan en la parte centro sur de la zona metropolitana, de ahí el mayor porcentaje de MI de las TDR; en cambio, como los lugares de bajos ingresos predominan en la periferia, las TDR tienen menos opciones 
de emplearse en su entorno próximo, lo que se refleja en el mayor número de desplazamientos o mayor porcentaje de ME. Precisamente el agrupamiento de municipios que se identificó en la parte oriente de la ZMCM se ubica en una zona que se caracteriza con tener predominantemente población de bajos ingresos y para quienes el costo del transporte público puede tener un peso importante en su economía.

\subsection{Las características sociodemográficas de dos patrones identificados}

A partir de lo anterior se seleccionaron los municipios según las agrupaciones reconocidas y se buscó identificar las diferencias entre dos delegaciones de la ciudad central (CC) y cuatro municipios de la zona oriente (ZO). Como se muestra en la Tabla 2, la variable espacial arroja diferentes resultados.

Zona Metropolitana Ciudad de México

Ciudad central

Movilidad

Externa

Interna

Situación conyugal

Unión libre

Casada

Soltera

Otros

Jefa

Esposa o pareja

Hija

Sin parentesco

Otros

\section{Nivel educativo}

Ninguno

Primaria

Secundaria

Preparatoria

Otros

4,7
95,3
00,00

100,00

6,3

17,8

56,5

19,4

100,0

11,2

6,8

3,2

75,0

3,7

100,0

5,0

43,2

37,7

8,4

5,7

100,0

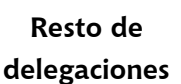

27,6

72,4

100,00

19,1

26,2

28,3

26,4

100,0

33,2

30,9

10,0

16,6

9,3

100,0

6,1

44,2

37,0

7,0

5,6

100,0
Municipios
conurbados

25,3

74,7

100,00

18,3

30,9

21,9

28,9

100,0

35,0

34,6

10,8

10,6

9,1

100,0

6,9

44,1

37,4

7,5

4,0

100,0
Zona oriente

60,6

39,4

100,00

22,6

28,8

14,4

34,2

100,0

39,8

39,9

12,6

0,5

7,2

100,0

6,3

50,1

34,2

7,0

2,4

100,0 


\begin{tabular}{lcccc} 
& \multicolumn{3}{c}{ Zona Metropolitana Ciudad de México } \\
Ciudad central & $\begin{array}{c}\text { Resto de } \\
\text { delegaciones }\end{array}$ & $\begin{array}{c}\text { Municipios } \\
\text { conurbados }\end{array}$ & Zona orient \\
Ingreso (salario mínimo mensual) & 0,2 & 0,2 & \\
Menos de uno & 6,3 & 15,9 & 19,9 & 14,1 \\
Uno & 17,5 & 39,0 & 41,1 & 29,2 \\
Dos & 34,3 & 26,9 & 21,2 & 7,6 \\
Tres & 41,6 & 18,0 & 17,6 & 7,3 \\
Más de tres & 100,0 & 100,0 & 100,0 & 100,0
\end{tabular}

Fuente: cálculos propios a partir de la Encuesta Intercensal 2015 del INEGI.

Primero destacan los porcentajes de movilidad externa de las TDR: la media de la zona metropolitana fue de 28,8\%; para la zona oriente, arrojó un $60,6 \%$ y para la ciudad central un $4,7 \%$ de ME. Es decir que más de la mitad de las TDR salen de la ZO a laborar a otro municipio; en contraste, la MI predomina en la ciudad central, con un $95,3 \%$.

Respecto a la ME de acuerdo con la situación conyugal, en la ZO los valores más altos se dan en las casadas, con un 30,5\%, y unión libre, con un $23,4 \%$; para la CC es un $24,4 \%$ solteras y un $23,5 \%$ casadas. $\mathrm{El}$ valor más alto de $\mathrm{MI}$ en la $\mathrm{CC}$ es de $58,2 \%$ en solteras. Al considerar la posición en el hogar, los valores altos se dan en jefa de familia, con 41,8 y $39,9 \%$ en la ZO y la CC respectivamente. El valor de MI a destacar en la CC es de $75 \%$ en la categoría sin parentesco.

En lo que se refiere a la edad, en la ZO la mayor proporción de movilidad externa se agrupa en el rango de 30 a 54 años, con un 71,6\%, y 17,3\% mayor de 55 años. Los valores más altos en la CC corresponden al rango de 40 a 64 años, con un $66,6 \%$, y un $17 \%$ mayor de 65 años. Así mismo, destaca el 6,3\% que pertenece al rango de 15 a 19 años en la CC, a diferencia de solo un $0,6 \%$ de este grupo en la ZO. En cuanto a la MI, en la CC el 57,7\% se ubica en el rango de 20 a 44 años. El nivel educativo presenta valores diferentes de ME en las dos zonas, en la ZO el valor más alto es de 52,8\% en nivel primaria, seguido del $34,5 \%$ en nivel secundaria. Para la CC, los valores corresponden a $38,2 \%$ en primaria y $31,9 \%$ en nivel secundaria.

Aunado al bajo nivel educativo, se suman los reducidos salarios que perciben: en este caso, la ME para la ZO presentó el valor más alto, con un $41,2 \%$ de trabajadores que perciben un salario mínimo mensual, y para la $\mathrm{CC}$, el 33,2\% percibe dos salarios mínimos mensuales. Para la movilidad 
interna los trabajadores presentaron en la $\mathrm{ZO}$ el $42,8 \%$ como valor más alto, que corresponde a un salario mínimo mensual, y para la $\mathrm{CC}$ el valor más alto fue de tres salarios mínimos mensuales, equivalentes al $34,7 \%$.

Pasando a las variables propiamente de movilidad, la ZO suma el $50 \%$ en los rangos de más de una hora y hasta dos horas (30,7\%), y más de 2 horas $(19,4 \%)$. Por su parte, la CC tiene el porcentaje de $58,9 \%$ en los TDR que no se trasladan. A su vez, el medio de transporte utilizado agrupa al 69,1\% de la ZO en combi o colectivo, y el 59,0\% de la CC no reporta ningún medio. De lo anterior se destaca la principal diferencia en la variable espacial: en la CC parece evidente que predomina la MI de los TDR con el tipo puertas adentro, los porcentajes altos indican que no invierten tiempo ni medio en trasladarse; esto, aunado a la categoría de $\sin p a-$ rentesco, permite asumir que viven en el lugar del empleador. Además, predomina un grupo de edad joven y soltero. Para el caso de la ZO prevalece la $\mathrm{ME}$, estos serían los trabajadores puertas afuera con viajes extremos o extreme commuting, su promedio de tiempo está entre hora y media y dos para trasladarse y es un grupo con una condición conyugal de casados y de edad mayor a 40 años.

\section{3. ¿A dónde se desplazan las TDR de la zona oriente?}

La teoría indica que los trabajadores se dirigen en mayor medida a municipios más cercanos al lugar de residencia que a otros más distantes (Susino y Martínez, 2010). Además, se considera que el grupo de ingresos más bajos trabaja principalmente en su entorno local y se asume que a menores recursos el desplazamiento será dentro del barrio, mientras que con mayores recursos los desplazamientos se darán a escala metropolitana (Jouffe, 2011).

Lo anterior no se cumple en un grupo de TDR que, aunque pueda parecer pequeño, representa la realidad de muchos trabajadores informales de la ciudad. El grupo de municipios de la ZO reporta una movilidad de los TDR a 22 diferentes municipios de la ZMCM, lo que implica que, además de los municipios cercanos, se desplazan a municipios más distantes. Como se aprecia en la Figura 3, las flechas indican el flujo de los desplazamientos de la ZO principalmente a las delegaciones centrales de la Ciudad de México: Coyoacán (1.807), Benito Juárez (1.448), Iztapalapa (1.080), Miguel Hidalgo (1.046) y Cuauhtémoc (1.030). 


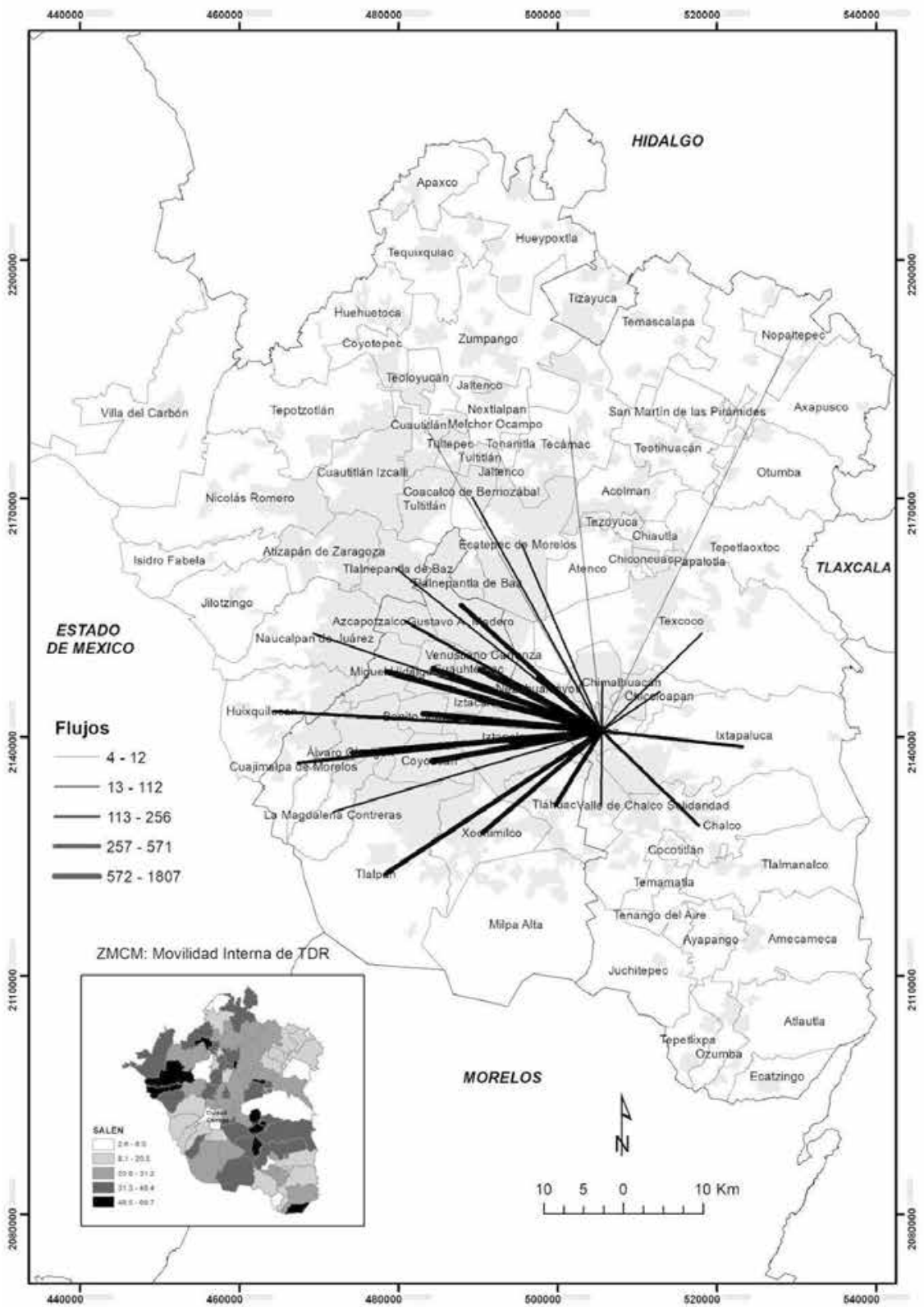

\section{FIGURA 3.}

ZMCM: flujos de movilidad de TDR de la zona oriente, 2015.

Fuente: cálculos propios a partir de la Encuesta Intercensal 2015 del INEGI.

Los desplazamientos de los TDR (ver Tabla 3) oscilaron, para el caso de Chimalhuacán, de una distancia mínima de $6 \mathrm{~km}$ al municipio colindante de La Paz hasta los $50 \mathrm{~km}$ lineales al municipio de Nopaltepec. En promedio la distancia de desplazamiento a los 27 diferentes municipios donde se ubica su lugar de trabajo es de $23 \mathrm{~km}$, con una desviación estándar de $11 \mathrm{~km}$. 
TABLA 3. DISTANCIAS MÍNIMAS Y MÁXIMAS DE ALGUNOS MUNICIPIOS HACIA SU MUNICIPIO DE TRABAJO

\begin{tabular}{llccccc} 
& & \multicolumn{5}{c}{ Distancia (lineales) } \\
& Mínima & N. ${ }^{\circ}$ de trabajadores & Municipio & Máxima & N. ${ }^{\circ}$ de trabajadores & Municipio \\
Chimalhuacán & $6 \mathrm{~km}$ & 47 & La Paz & $50 \mathrm{~km}$ & 4 & Nopaltepec \\
La Paz & $6 \mathrm{~km}$ & 43 & Chimalhuacán & $41 \mathrm{~km}$ & 94 & Huixquilucan \\
Chicoloapan & $7 \mathrm{~km}$ & 69 & Chimalhuacán & $48 \mathrm{~km}$ & 5 & Huixquilucan \\
Valle de Chalco & $7 \mathrm{~km}$ & 261 & Tlahuac & $38 \mathrm{~km}$ & 22 & Cuajimalpa \\
Nicolás Romero & $14 \mathrm{~km}$ & 923 & Atizapán & $46 \mathrm{~km}$ & 26 & Nezahualcoyotl
\end{tabular}

Fuente: cálculos propios a partir de información vectorial.

El número de TDR que se desplaza de los cuatro municipios de la zona oriente representa el 5,9\% del total de TDR de la zona metropolitana. Son trabajadores que, además de tener un trabajo precario, residen en una zona con alto índice de pobreza, tienen bajo nivel de escolaridad e ingresos de hasta un salario mínimo, son mujeres en su mayoría y deciden realizar largos desplazamientos para emplearse, integrándose así a las oportunidades que encuentran en la ciudad.

\subsection{Saldos de movilidad}

De forma general, y tomando en cuenta toda la ZMCM, el análisis se puede completar si se consideran los municipios, a la vez, como lugares de origen y de destino de los desplazamientos. Al comparar ambas variables obtenemos los saldos entre las salidas y las entradas en cada municipio.

Del total de municipios de la zona metropolitana, el $76 \%$ tiene saldos negativos y presenta una distribución más dispersa, a diferencia de los saldos positivos, que corresponden a 18 municipios, con una mayor concentración en el centro. Básicamente, 58 de los 76 municipios son expulsores de trabajadores, es decir que salieron más trabajadores a desarrollar su actividad a otros municipios de los que entraron a trabajar, lo que supone un saldo negativo.
Entre los 18 municipios receptores se destaca Coyoacán, cuyo saldo positivo equivale a 6.630 trabajadores. A continuación se sitúan Benito Juárez, con 5.880, y Miguel Hidalgo, con 4.826. En el lado opuesto se encuentran los municipios con mayor déficit, Chimalhuacán, Iztapalapa y Nicolás Romero, con $-6.142,-4.503$ y -4.332 respectivamente.

Considerando los principales municipios de origen y destino de los desplazamientos, se puede identificar que los municipios de destino tienen un porcentaje de pobreza menor del $35 \%$, que incluyen Benito Juárez y Miguel Hidalgo, con el 8 y el $14 \%$ de porcentaje de pobreza. Los municipios de origen tienen un porcentaje superior al $35 \%$, que incluye a los municipios de Valle de Chalco y Chimalhuacán, con el 58 y el $62 \%$ de porcentaje de pobreza respectivamente.

A partir de lo anterior se puede señalar que a medida que aumenta el porcentaje de pobreza, en este caso, después del $35 \%$, se identifica una tendencia a ser municipios de origen de los desplazamientos para trabajar en el servicio doméstico, y, por el contrario, al disminuir el porcentaje de pobreza los municipios tienden a ser destino de los desplazamientos. Dicha relación es lógica si se considera que para los TDR la clase media y media alta es su principal fuente de empleo, entonces, mientras menor sea el porcentaje de pobreza en un municipio, mayor posibilidad de localización de clases medias. 
Es así que el patrón origen/destino de los desplazamientos de los TDR guarda una estrecha relación con el patrón de distribución de la población que caracteriza la zona metropolitana, con la población de mayor ingreso cerca del centro principal y los pobres en su periferia.

\section{Conclusiones}

La expansión urbana de la ciudad conformó áreas en la periferia con carencia de servicios urbanos básicos y una deficiente infraestructura de transporte, zonas que se caracterizan por concentrar población de bajos ingresos, principalmente al oriente de la zona metropolitana. Esta segregación funcional genera una dependencia de la población respecto al centro de la ciudad, de modo que la población que no se integra al mercado laboral de su lugar de residencia tiene que desplazarse a otras zonas de la ciudad, condición que es más grave para la población que se emplea en ocupaciones informales y en especial para las mujeres.

Se hizo evidente que la segregación urbana de la ZMCM determina la modalidad de movilidad de las mujeres TDR, se identificó un contraste en la MI que predomina en la CC y una alta $\mathrm{ME}$ en los municipios de la $\mathrm{ZO}$ y norponiente de la periferia. Las TDR que se desplazan de los municipios de alta ME se ubican en una zona de la periferia con alto grado de pobreza, reciben en promedio un salario mínimo mensual de ingreso y reportan tiempos de traslado de una y hasta dos horas de duración, lo que hace probable que sus desplazamientos impliquen condiciones de transporte incómodas y altos costos monetarios.

Por otro lado, la proporción alta de MI que tiene una concentración destacada en las delegaciones de la ciudad central, particularmente Miguel Hidalgo y Benito Juárez, implica una mayor inclusión al acceder a las ventajas de residir en lugares con mejor infraestructura y servicios. Para el caso de las delegaciones centrales mencionadas, dados los resultados de las variables censales utilizadas, se asume que predomina la modalidad de empleo tradicional de este grupo de trabajo, es decir, el trabajo puertas adentro o las trabajadoras que residen en el mismo lugar del empleador.

En cuanto al alto desplazamiento de trabajadores de los municipios periféricos, esto puede implicar una ventaja que permite evitar, con su movilidad, el aislamiento o la exclusión que se daría si trabajan en el mismo lugar de residencia, en este caso, una periferia pobre y segregada, con pocas oportunidades y servicios. Como señala Jirón (2009, p. 88), "si la localización periférica de vivienda genera y refuerza la segregación, la mezcla de población puede no ser la única solución; la movilidad diaria puede ser vista como una posibilidad de disminuir o aumentar la segregación al abrir puertas de entrada a nuevas y diferentes posibilidades de apropiación de lugares".

Resumiendo, las TDR y en general los grupos sociales de bajos ingresos tienen en la movilidad cotidiana el principal y quizá único medio para resolver o minimizar la desigualdad de acceso a las oportunidades. Esto es importante para estos grupos, que acumulan desventajas: la desventaja social y educativa, la desventaja espacial, si residen en una zona segregada, la desventaja del empleo precario o informal y la desventaja de una movilidad limitada por la falta de acceso a un buen servicio de transporte. Esta situación los pone, según sea el caso, en riesgo de entrar a una condición de pobreza o permanecer en ella. 


\section{Referencias}

Aguilar, A. G., Romero, P. y Hernández, J. (2015). Segregación socio-residencial en la Ciudad de México. Dinámica del patrón territorial a nivel local, 2000-2010. En A.G. Aguilar e I. Escamilla (coords.), Segregación urbana y espacios de exclusión. Ejemplos de México y América Latina (pp. 73-102). Serie Estudios Urbanos Porrúa, UNAM. México: Instituto de Geografía, UNAM.

Avellaneda, P. (2008). Movilidad cotidiana, pobreza y exclusión social en la ciudad de Lima. Anales de Geografía, 28(2), 9-35.

Avellaneda, P. (2009). La investigación cualitativa en el estudio de las relaciones entre movilidad cotidiana y pobreza en el contexto latinoamericano. Un caso aplicado en la Lima metropolitana. Documentos de Anales Geográficos, 55, 57-76.

Barrios, M. C. y Hernández, M. (2014). Análisis sociodemográfico y espacial de la movilidad laboral diaria en Canarias. Boletín de la Asociación de Geógrafos Españoles, 66, 7-24.

Blumen, O. (1994). Gender differences in the journey to work. Urban Geography, 15, 223-245.

Carrión, F. (2003). Centros históricos y pobreza en América Latina. [Mimeo]. Banco Interamericano de Desarrollo. Recuperado de http://www.flacso.org.ec/docs/ fc_centrohisto.pdf.

Casado, J. M. (2000). Diferencias de género en los desplazamientos cotidianos por razones laborales. [Documento de trabajo del IVIE WP-EC-2000-03]. Valencia: Instituto Valenciano de Investigaciones Económicas.

Díaz, M. Á. (1989). Movilidad femenina en la ciudad. Notas a partir de un caso. Documents d'Anàlisi Geogràfica, 14, 219-239.

Díaz, M. Á. (1995). Género y estructura urbana en los países occidentales. Anales de Geografía de la Universidad Complutense, 15, 267-279.

Duhau, E. y Giglia, A. (2008). Las reglas del desorden: habitar la metrópoli. México D. F.: Siglo XXI.

Consejo Nacional de Evaluación de la Política de Desarrollo Social (Coneval). (2010). Pobreza a nivel municipio 2010, México. México: Coneval.

Figueroa, M. C. y Waintrub, S. N. (2015). Movilidad femenina en Santiago de Chile: reproducción de inequidades en la metrópolis, el barrio y el espacio público. Urbe. Revista Brasileira de Gestão Urbana, 7(1), 48-61.

García, J. H., Tomaseti, E. y Sánchez F. J. (2013). Una aplicación del análisis exploratorio de datos espaciales (AEDE) con GeoDa en el fracaso empresarial en España. Jornadas de introducción a la investigación de la UPCT, 6, 145-147.

Gielen, E. (2016). Costes del "Urban Sprawl" para la Administración local: el caso valenciano. Valencia: Servicio de Publicaciones de la Universitat de Valencia.

Graizbord, B. y Acuña, B. (2007). Movilidad residencial en la Ciudad de México. Estudios Demográficos y Urbanos, 11(2), 291-335. 
Ibarra, V. y Escamilla, I. (2016). La geografía feminista, de género y de la sexualidad en México, un saber en crecimiento. En V. Ibarra e I. Escamilla (coords.), Geografías feministas de diversas latitudes. Orígenes, desarrollo y temáticas contemporáneas (pp. 209-238). México: Instituto de Geografía, UNAM.

Instituto Nacional de Estadística y Geografía (INEGI). (2012). Perfil sociodemográfico de los trabajadores domésticos remunerados en México 2010. México: INEGI.

Instituto Nacional de Estadística y Geografía (INEGI) (2015a). Encuesta Intercensal 2015. Base de datos de la muestra. Aguascalientes: INEGI.

Instituto Nacional de Estadística Geografía e Informática (INEGI). (2015b). Encuesta Nacional de Ocupación y Empleo 2015. Aguascalientes: INEGI.

Jaramillo, S. (1999). El papel del mercado del suelo en la configuración de algunos rasgos socioespaciales de las ciudades latinoamericanas. Revista Territorios, 2, 107-129.

Jirón, P. (2009). Prácticas de movilidad cotidiana urbana: un análisis para revelar desigualdades en la ciudad. En M. Tironi y F. Pérez (coords.), Espacios, prácticas y cultura urbana (pp. 176-189). Santiago de Chile: ARQ Ediciones, Escuela de Arquitectura, Universidad Católica de Chile.

Jirón, P. y Zunino, S. D. (2017) Dossier. Movilidad urbana y género: experiencias latinoamericanas. Revista Transporte y Territorio, 16, 1-8.

Jouffe, Y. (2011). Las clases socio-territoriales entre movilidad metropolitana y repliegue barrial. ¿Tienen los pobladores pobres una movilidad urbana de clase? Revista Transporte y Territorio, 4, 84-116.

Lazo, A., y Contreras, Y. (2009). Aproximación exploratoria al estudio de la movilidad cotidiana de las mujeres. El caso de La Pintana. Santiago de Chile. Ponencia presentada en el $12^{\circ}$ Encuentro de Geógrafos de América Latina, 3-7 de abril, Montevideo, Uruguay.

López, E. (2015). Suelo urbano y segregación residencial: hacia una agenda de integración social para zonas centrales metropolitanas chilenas. Ciudades, 18(1), 197-213.

Miralles-Guasch, C. y Cebollada, A. (2009). Movilidad cotidiana y sostenibilidad, una interpretación desde la geografía humana. Boletín de la Asociación de Geógrafos Españoles (AGE), 50, 193-216.

Miralles-Guasch, C. y Martínez-Melo, M. (2012). Las divergencias de género en las pautas de movilidad en Cataluña, según edad y tamaño del municipio. Revista Latino-Americana de Geografia e Gênero, Ponta Grossa, 3(2), 49-60.

Melo, E. C y De Freitas, T. A. (2010) La distribución espacial y auto-correlación de indicadores de la salud de la madre y los indicadores de salud en el estado de Paraná, Brasil. Revista Latino-Americana de Enfermagem, 18(6), 1177-86. Recuperado de http://www.scielo.br/pdf/rlae/v18n6/es_19.pdf.

Módenes, J. A. (2008) Movilidad espacial, habitantes y lugares: retos conceptuales y metodológicos para la geodemografía. Estudios Geográficos, 69(264), 157-178. 
Moreno, C. y Rubiano, M. (2014). Segregación residencial y movilidad cotidiana en el contexto metropolitano. Un estudio a partir de las relaciones Bogotá-Soacha. Revista Territorios, 31,133-162.

Moreno, J. y Partida, V. (2011), Factores que afectan la expansión urbana del noreste de la Zona Metropolitana del Valle de México (2000-2005). Clío América, 5(9), 12-49.

Moovit. (2016). Informe de uso global de transporte público en las ciudades 2016. Recuperado de https://www.company.moovitapp.com/public-transit-usagereport-2016.

Organización Internacional del Trabajo (OIT). (2013). Panorama laboral 2012. América Latina y el Caribe. Lima: OIT.

Pírez, P. (2013). La urbanización y la política de los servicios urbanos en América Latina. Andamios. Revista de Investigación Social, 10(22), 45-67.

Pradilla, C. E. (2015). De la ciudad compacta a la periferia dispersa. Ciudades, 106, 2.

Rodgers, J. (2009). Cambios en el servicio doméstico en América Latina. En C. Mora y M. E. Valenzuela (eds.), Trabajo doméstico: un largo camino hacia el trabajo decente (pp. 71-114). Santiago de Chile: OIT.

Rodríguez, J. y García, J. (2012). Diversidad de género en la movilidad cotidiana en la comunidad de Madrid. Boletín de la Asociación de Geógrafos Españoles, 58, 105131.

Siscar, M. (23 de junio 2015) La distribución del ingreso, cuestión de vértigo. Animal Político. Recuperado de https://pajaropolitico.com/tu-sueldo-frente-a-ladesigualdad/2015/06/23/la-distribucion-del-ingreso-cuestion-de-vertigo/.

Soto, V. P. (2017). Diferencias de género en la movilidad urbana. Las experiencias de viaje de mujeres en el Metro de la Ciudad de México. Revista Transporte y Territorio, 16, 127-146.

Susino, J. y Duque, R. (2013) Veinte años de suburbanización en España (1981-2001). El perfil de sus protagonistas. Documents d'Anàlisi Geogràfica, 59(2), 265-290.

Susino, J. y Martínez J. (2010). Movilidad cotidiana por trabajo en el análisis de la estructura territorial: Andalucía y Centro de México. Papeles de Población, 16(66), 137-176.

Susino, J. y Torrado, J. M. (2015). Características y condiciones de la movilidad cotidiana. Sevilla: Consejería de Fomento y Vivienda, Junta de Andalucía.

Vega, A. L. (2013, marzo). La urbanización en la periferia de la Ciudad de México. En: Memorias del Congreso Nacional de Vivienda. Programa Universitario de Estudios sobre la Ciudad (pp. 221-245). México: UNAM.

Vega, Ana Lourdes. (2015). La urbanización en la periferia de la Ciudad de México. En A. Ziccardi y A. González (Coords.), Habitabilidad y política de vivienda en México (pp. 275-294). México: PUEC.

Valdés, S. C. (2008). La movilidad en la Comunidad de Madrid: ¿una cuestión de género? [Monografía]. Madrid: Universidad Politécnica de Madrid. 
